\title{
Light-Mediated Collective Atomic Motion in an Optical Lattice Coupled to a Membrane
}

\author{
Aline Vochezer, ${ }^{1, *}$ Tobias Kampschulte, ${ }^{1, \dagger}$ Klemens Hammerer, ${ }^{2}$ and Philipp Treutlein ${ }^{1, \$}$ \\ ${ }^{1}$ Department of Physics, University of Basel, Klingelbergstrasse 82, 4056 Basel, Switzerland \\ ${ }^{2}$ Institute for Theoretical Physics and Institute for Gravitational Physics (Albert Einstein Institute), \\ Leibnitz University Hannover, Callinstrasse 38, 30167 Hannover, Germany
}

(Received 24 May 2017; published 14 February 2018)

\begin{abstract}
We observe effects of collective atomic motion in a one-dimensional optical lattice coupled to an optomechanical system. In this hybrid atom-optomechanical system, the lattice light generates a coupling between the lattice atoms as well as between atoms and a micromechanical membrane oscillator. For large atom numbers we observe an instability in the coupled system, resulting in large-amplitude atommembrane oscillations. We show that this behavior can be explained by light-mediated collective atomic motion in the lattice, which arises for large atom numbers, small atom-light detunings, and asymmetric pumping of the lattice, in agreement with previous theoretical work. The model connects the optomechanical instability to a phase delay in the global atomic backaction onto the lattice light, which we observe in a direct measurement.
\end{abstract}

DOI: 10.1103/PhysRevLett.120.073602

Ultracold atoms in optical lattice potentials formed by the interference of laser beams are a powerful system for many-body physics [1], quantum information science [2], and precision metrology [3]. In most optical lattice experiments the lattice light is far detuned from any atomic resonance, providing a conservative external potential with negligible backaction of the atoms onto the lattice light. The backaction is significantly enhanced when operating at moderate atom-light detuning in the tens of $\mathrm{MHz}$ to $\mathrm{GHz}$ regime [4-6] and at high atomic density. In this regime, the lattice light can mediate long-range interactions that couple the motion of atoms in different lattice potential wells. These interactions have been predicted to give rise to a variety of intriguing phenomena, ranging from spontaneous self-ordering and crystallization of light and atoms $[7,8]$ to the appearance of traveling wavelike collective oscillations of the atoms that can destabilize the entire lattice $[9,10]$. Moreover, the backaction onto the lattice light can also be exploited to dynamically couple the atoms to other physical systems such as micromechanical oscillators [11-15]. Such hybrid atom-optomechanical systems offer new perspectives for ground-state cooling and quantum control of engineered mechanical structures [16-21] and for studies of nonequilibrium quantum phase transitions [22].

In the experiments reported here we observe effects of light-mediated collective atomic motion in an optical lattice in the context of building a hybrid system where the lattice light couples the atoms to a micromechanical membrane oscillator. The membrane acts like an additional "superatom" of particularly high polarizability, enhancing collective effects in the entire system and providing a convenient way to directly detect the dynamics of the coupled system. For large numbers of atoms in the lattice we observe a dynamic instability of the coupled system, which can be explained in a model that takes light-mediated interactions of the atoms into account. These long-range atomic interactions also lead to an additional phase delay in the global atomic backaction onto the light field, which we observe in experiments. The phase delay can induce unstable behavior in the hybrid system if the atommembrane coupling is large, even if the lattice itself is still stable. Our experiments show that light-mediated atom-atom interactions are significant even in free-space optical lattices, providing a way to study nonequilibrium many-body physics $[7-10,22]$ that is complementary to experiments with atoms in optical cavities [23].

Our hybrid system is illustrated in Fig. 1(a). It consists of a $\mathrm{Si}_{3} \mathrm{~N}_{4}$ membrane oscillator with mass $M=117 \mathrm{ng}$ and vibration frequency $\Omega_{m}=2 \pi \times 276 \mathrm{kHz}$ in an optical cavity [24] and an ensemble of $N$ ultracold Rubidium atoms of mass $m$ in an optical lattice. The lattice is generated by a laser beam that also drives the membrane

(a)

(b)

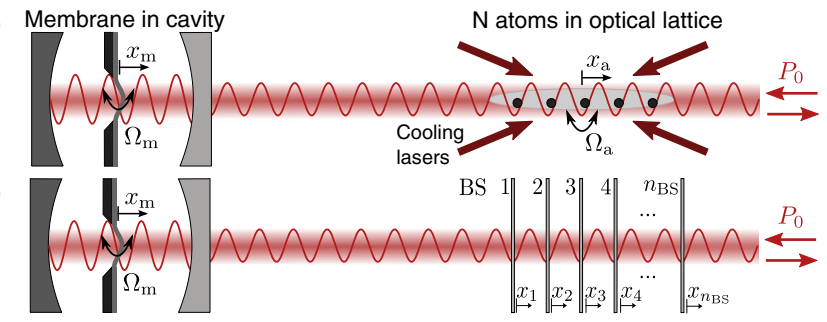

FIG. 1. (a) Optomechanical coupling scheme of atoms in an optical lattice and a micromechanical membrane oscillator in an optical cavity. (b) Modeling the atoms as beam splitters (BSs) allows us to describe light-mediated collective motion of the atoms (see text). 
cavity with a detuning $\Delta$ much smaller than the cavity linewidth $\kappa$ [25]. For large frequency detunings $\Delta_{\mathrm{LA}}$ of the driving laser from the atomic transition and small atomic densities, light-mediated atom-atom interactions are negligible and the atoms oscillate with a frequency $\Omega_{a} \propto \sqrt{P_{0}}$ in the lattice potential wells, adjustable via the driving laser power $P_{0}$ [26]. As predicted in [16-18] and observed in $[13,14]$, radiation-pressure forces mediated by the lattice light couple the vibrations of atoms and membrane over a large distance. In the absence of collective atomic effects all atoms couple equally to the membrane, resulting in a linear coupling of the membrane displacement $x_{m}$ to the atomic center of mass displacement $x_{a}$ with a coupling constant $g_{N}=\left|r_{m}\right| \Omega_{a} \sqrt{N m \Omega_{a} / M \Omega_{m}}(2 F / \pi)$, where $F=570$ is the cavity finesse and $r_{m}=0.41$ the membrane reflectivity $[16,17]$. The coupling mechanism exploits the fact that a displacement of the membrane induces a phase shift of the reflected light, which displaces the lattice potential wells. Conversely, a displacement of the atoms changes the optical power traveling towards the membrane and with this the radiation pressure force on the membrane. Additional cooling lasers applied to the atoms result in strong damping of the atomic motion at a rate $\Gamma_{a} \gg g_{N}$ and cool the atomic cloud to $4 \mathrm{mK}$. In the absence of atom-atom interactions the coupling then leads to sympathetic cooling of the membrane vibrations at a rate $\Gamma_{\mathrm{sym}}=4 \eta^{2} t^{2} g_{N}^{2} / \Gamma_{a}$ (for resonant coupling $\Omega_{a}=\Omega_{m}$ ). Here $\eta \approx 1$ is the incoupling efficiency into the optical cavity and $t=0.71$ the amplitude transmission of the optical path between atoms and membrane $[13,14,17]$. This has been used in the experiments of Ref. [14] to cool a membrane oscillator from room temperature to $0.7 \mathrm{~K}$ using the atoms as coolant.

The sympathetic cooling measurements of Ref. [14] were performed with large light-atom detuning $\Delta_{\mathrm{LA}}=$ $-2 \pi \times 8 \mathrm{GHz}$ from the $F=2 \leftrightarrow F^{\prime}=3$ transition of the ${ }^{87} \mathrm{Rb} D_{2}$ line at $\lambda=780 \mathrm{~nm}$. Here we focus on small $\Delta_{\mathrm{LA}} \approx-2 \pi \times 1 \mathrm{GHz}$ where the coupled dynamics becomes drastically different. An instability occurs at large atom numbers, where the membrane amplitude starts to grow exponentially because the total membrane damping rate $\Gamma_{\text {tot }}=\Gamma_{m}+\Gamma_{\text {opt }}+\Gamma_{\text {sym }}$ changes sign from positive to negative. In $\Gamma_{\text {tot }}$ we include the intrinsic membrane damping rate $\Gamma_{m}=0.96 \mathrm{~s}^{-1}$ and standard cavity-optomechanical damping at rate $\Gamma_{\mathrm{opt}}=10.6 \mathrm{~s}^{-1}$ [27], which arises from the small red laser-cavity detuning $\Delta=-0.06 \kappa$. We observe this instability in experiments where we detect the membrane amplitude with an additional detection beam and vary the number of atoms in the lattice volume $N_{\text {lat }}$ [25]. The number of resonantly coupled atoms $N$ is smaller than $N_{\text {lat }}$ because of the inhomogeneous transverse lattice profile. As in [14], we estimate $N=\left[\left(\pi \Gamma_{a}\right) / 2 \Omega_{m}\right] N_{\text {lat }}$. During the preparation of the atomic ensemble the lattice is operating at low driving laser power $P_{0}=0.1 \mathrm{~mW}$ and the atoms do not couple to the membrane because $\Omega_{a} \ll \Omega_{m}$. At the start of the coupling experiment the lattice is ramped up in $10 \mathrm{~ms}$ to $P_{0}=3.4 \mathrm{~mW}$, which tunes the atomic oscillation frequency into resonance with the membrane frequency. The red traces in Fig. 2(a) exemplarily show how the mean square membrane displacement $\left\langle x_{m}^{2}(t)\right\rangle$ subsequently evolves with time for different $N_{\text {lat }}$. For the smallest $N_{\text {lat }}$ we observe strong damping of the membrane motion resulting in a steady state value below the optomechanical cooling level (blue curve). This is the sympathetic cooling effect observed in [14]. Subsequent atom loss from the ensemble on the time scale of several seconds reduces the cooling effect as expected from $\Gamma_{\text {sym }} \sim N$. For the next larger $N_{\text {lat }}$ the membrane amplitude decreases only after some atoms have been lost. For even larger $N_{\text {lat }}$ the system becomes unstable. Now the membrane amplitude increases after the turn-on and performs limit cycle oscillations at a large amplitude $\left\langle x_{m}^{2}(t)\right\rangle /\left\langle x_{m, t h}^{2}(t)\right\rangle \approx 100$ before it slowly
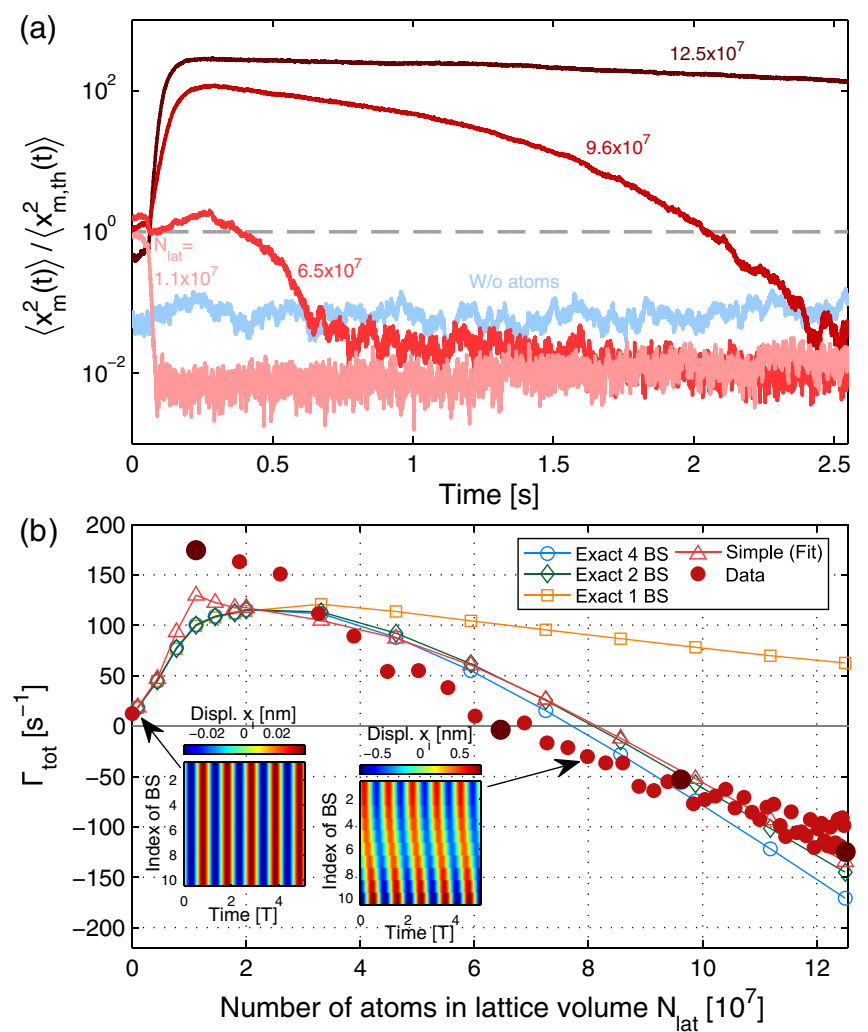

FIG. 2. Observation of self-oscillations. (a) Evolution of $\left\langle x_{m}^{2}(t)\right\rangle$ with time for different $N_{\text {lat }}$. Red traces: Lattice is ramped up at $t=50 \mathrm{~ms}$. Blue trace: Lattice is running at $P_{0}=3.4 \mathrm{~mW}$ continuously with $N_{\text {lat }}=0$. Dashed gray: Room temperature level. (b) Total membrane damping rate $\Gamma_{\text {tot }}$ versus $N_{\text {lat }}$. Filled red circles: Data extracted from traces as in (a). Larger red circles: Data points of traces in (a). Empty blue circles: Numerical simulation with exact model with four beam splitters (BS). Empty green diamonds: As blue, but with 2 BS. Empty orange squares: As blue and green, but with only 1 BS. Empty red triangles: Simulation of linearized model with 2 BS fitted to the data. For all four curves, $\Gamma_{a}=233 \mathrm{~s}^{-1}$ and $\alpha=0.11$. Insets: Simulated displacement $x_{i}$ of an array of $10 \mathrm{BS}$ as a function of time for $0.3 \times 10^{7}$ (left) and $8 \times 10^{7}$ (right) atoms. 
decreases when atoms are lost. Here $\left\langle x_{m, t h}^{2}(t)\right\rangle^{1 / 2}$ is the room temperature thermal amplitude of the membrane. Thus, we find that at large atom number the presence of the atoms induces an instability, corresponding to negative $\Gamma_{\text {tot }}$. This behavior is not predicted by previous theoretical descriptions of the atom-membrane coupling [16,17], which neglect collective atomic effects. From the slopes of $\left\langle x_{m}^{2}(t)\right\rangle$ after the turn-on of the lattice, we can extract $\Gamma_{\text {tot }}$, which is plotted in Fig. 2(b) against $N_{\text {lat }}$ (filled red circles).

We can model the observed behavior if we take lightmediated interactions between the atoms in different lattice wells into account. Following [10], we model the combined effect of the $N_{\text {BS }}$ atoms in each potential well as a thin BS as illustrated in Fig. 1(b) with reflection and transmission coefficients given by the dimensionless atomic polarizability density $\zeta=\left[\Gamma /\left(-\Delta_{\mathrm{LA}}\right)\right]\left[N_{\mathrm{BS}} \lambda^{2} /\left(4 \pi \sigma_{L}\right)\right]$, where $\Gamma$ is the natural linewidth of the atomic transition and $\sigma_{L}$ the transverse area of the laser beam. The imaginary part of $\zeta$ is omitted since $\left|\Delta_{\mathrm{LA}}\right| \gg \Gamma$. We calculate the forces on the $n_{\mathrm{BS}}=N / N_{\mathrm{BS}}$ atomic BSs and the membrane using the transfer matrix method. The detailed model is presented in [25], where we apply the theory of [10] to our system. With this model we perform numerical simulations of the coupled dynamics and extract a theoretical value for $\Gamma_{\text {tot }}$. The model predicts the instability at large $N$ and that anharmonicities in the atomic potential stop the exponential growth of the membrane amplitude and stabilize the limit cycle oscillation.

The blue (green) circles (diamonds) in Fig. 2(b) show the result of numerical simulations with the atoms distributed over four (two) atomic BSs. The red triangles are the result of an analytical analysis (see below) and the orange squares show the damping rate for one atomic BS, i.e. without collective effects. The numerical simulations have been performed for a reasonable $\Gamma_{a}=233 \mathrm{~s}^{-1}$, and $N_{\text {lat }}$ has been scaled by a factor $\alpha=0.11$ for the simulation to match the data. This is plausible as the temperature of the atomic cloud $(\approx 4 \mathrm{mK})$ is larger than the depth of the coupling lattice $(\approx 500 \mu \mathrm{K})$ so that not all atoms are trapped. Both traces (blue and green) do not exactly reproduce the data. This can be due to the fact that the model of the atomic ensemble is greatly simplified. As we operate the system when the lattice is overlapped with a large magneto-optical trap [25], we do not have direct access to the atoms taking part in the coupling. However, the main features such as the initial linear increase of $\Gamma_{\text {tot }}$ and the subsequent decrease leading to negative damping are confirmed by the model and the numbers match roughly. If we replace the membrane by a fixed mirror, we also simulate unstable behavior as in $[9,10]$ but for larger $N_{\text {lat }}$.

The traces with four and two BSs differ only slightly, whereas the simulation with only one atomic BS (orange squares) does not show the instability [28] indicating that coupled motion of atoms in different lattice wells plays an essential role. We observe that the behavior quickly converges for more than two BSs suggesting that only a few collective atomic modes are relevant. The insets in Fig. 2(b) show exemplarily how the displacements $x_{i}$ of ten BSs evolve as a function of time for $0.3 \times 10^{7}$ atoms and $8 \times 10^{7}$ atoms. For the small atom number all BSs move in phase and do not interact so that only their center of mass motion couples to the membrane. For the large atom number a traveling wavelike collective oscillation appears. In this case more than one collective atomic mode must take part in the coupling. Given the fast convergence for more than two BSs we have a closer look at the simplest model, the membrane coupled to a stack of two BSs. For this two-BS model we linearize the radiation pressure forces around the steady state positions of the BSs and the membrane and expand the linear coefficients up to third order in $\zeta$. This model enables us to describe the onset of instability, but does not cover the regime of limit cycles. We find the following linear equations of motion for the displacement of the membrane $x_{m}$ and the two atomic BSs $x_{1}$ and $x_{2}$ :

$$
\begin{aligned}
\ddot{x}_{m} & =-\Gamma_{m}^{\prime} \dot{x}_{m}-\Omega_{m}^{2} x_{m}+k_{m m} x_{m}+k_{m 1} x_{1}+k_{m 2} x_{2}, \\
\ddot{x}_{1} & =-\Gamma_{a} \dot{x}_{1}+k_{1 m} x_{m}+k_{11} x_{1}+k_{12} x_{2}, \\
\ddot{x}_{2} & =-\Gamma_{a} \dot{x}_{2}+k_{2 m} x_{m}+k_{21} x_{1}+k_{22} x_{2},
\end{aligned}
$$

with $\Gamma_{m}^{\prime}=\Gamma_{m}+\Gamma_{\mathrm{opt}}$ and coefficients

$$
\begin{array}{rlrl}
k_{m m} & =\frac{N m}{2 M} \Omega_{a}^{2} R(-2+10 \nu) f^{2}, \\
k_{m 1} & =\frac{N m}{2 M} \Omega_{a}^{2} R(1-9 \nu) f, \\
k_{m 2} & =\frac{N m}{2 M} \Omega_{a}^{2} R(1-\nu) f, \\
k_{1 m} & =\Omega_{a}^{2}(1-\nu) f, & k_{2 m} & =\Omega_{a}^{2}(1-9 \nu) f, \\
k_{11} & =\Omega_{a}^{2}(-1+\nu), & k_{21} & =\Omega_{a}^{2} 8 \nu, \\
k_{12} & =0, & k_{22} & =\Omega_{a}^{2}(-1+\nu) .
\end{array}
$$

Here $R=\eta t^{2}$ is the lattice amplitude reflection coefficient, $f=2\left|r_{m}\right|[(2 F) / \pi]$ the cavity enhancement factor, and $\nu=\left[\left(\mathcal{A}^{2} \zeta^{2}\right) / 8\right]$ a dimensionless parameter that depends on the polarizability density $\zeta \propto N$ of a single atomic $\mathrm{BS}$ and the lattice asymmetry $\mathcal{A}=\left(1-R^{2}\right) / R$. The parameter $\nu$ describes the effect of collective atomic motion in leading order of $\zeta$, and $\nu=0$ recovers the case of noninteracting atoms. In the experiments $\mathcal{A}$ is fixed, so that $\nu$ scales with $N$. A numerical simulation of these simplified equations of motion [red triangles in Fig. 2(b)] reproduces the exact result with two BS (green diamonds) as expected. For $\nu \ll 1$, i.e. small $N$, both BSs couple to the membrane equally and move independently of each other. We can then rewrite the equations of motion as a coupling between the membrane displacement $x_{m}$ and the atomic center of mass 
displacement $x_{a}=\left(x_{1}+x_{2}\right) / 2$ reproducing the result of Refs. [14,17]. However, for larger $N$ (large $\nu$ ) the atoms interact with each other and the membrane does not couple to a single atomic mode any more. Note that the dynamics described by the set of Eqs. (1) is nonconservative $\left(k_{21} \neq k_{12}=0\right)$, a consequence of the cascaded nature of the system.

The instability can also be understood in a feedback picture: The coupled atom-membrane motion becomes unstable if a signal traveling from the membrane to the atoms and back experiences a phase delay of $360^{\circ}$ and a loop gain larger than unity [29]. A system of only two coupled harmonic oscillators, such as the membrane coupled to one atomic oscillator (e.g., the atomic center of mass motion), cannot become unstable as the maximum phase delay collected in one round trip stays below $2 \times 180^{\circ}=360^{\circ}$. If an additional harmonic oscillator, e.g., in form of a second collective atomic mode, takes part in the coupling, the atomic backaction onto the laser beam traveling towards the membrane can be delayed by more than $180^{\circ}$, providing a necessary condition for instability of the coupled system.

To directly observe this phase delay, we performed experiments in which the phase shift induced by the membrane was mimicked by a fiber electro-optic modulator $(\mathrm{EOM})$ and the atomic backaction onto the lattice power was detected with a photodiode as depicted in the inset of Fig. 3(a) [25]. Figures 3(a) and 3(b) show how amplitude and phase of the photodiode signal evolve as a function of the EOM modulation frequency $\Omega$ for different $N_{\text {lat }}$. The dashed (dashed-dotted) traces show the expected behavior for the one-BS model used in Refs. [14,17] (the two-BS model); see [25]. The one-BS model predicts a maximum phase delay of $180^{\circ}$. In contrast, for large atom numbers the data and the two-BS model show phase delays $>180^{\circ}$ indicating that the one-BS model is not sufficient to describe the system and showing that the coupled atom-membrane motion can indeed become unstable. For the theory curves the inhomogeneously broadened atomic ensemble has been modeled with all atoms $\left(N=N_{\text {lat }}\right)$ and an increased, inhomogeneously broadened atomic linewidth $\Gamma_{a}$ [25] in contrast to Fig. 2(b) where only the resonant atoms are taken into account. Insufficient knowledge of the exact properties of the atoms in the lattice makes a more precise modeling of the atomic backaction difficult. The great simplification in modeling the atomic ensemble as well as uncertainties in the signal calibration lead to a discrepancy in the signal amplitude heights between data and theory. Still, the oneand two-BS model show a drastic difference in the phase behavior for realistic parameters.

An additional phase delay enters into our system from the propagation time delay between atoms and membrane $\tau_{\text {prop }}=30 \mathrm{~ns}$ and the finite response time of the cavity $\tau_{\text {cav }}=0.6$ ns. However, these delays are of minor importance for the stability of our system, which was confirmed by varying the path length between atoms and membrane [25].
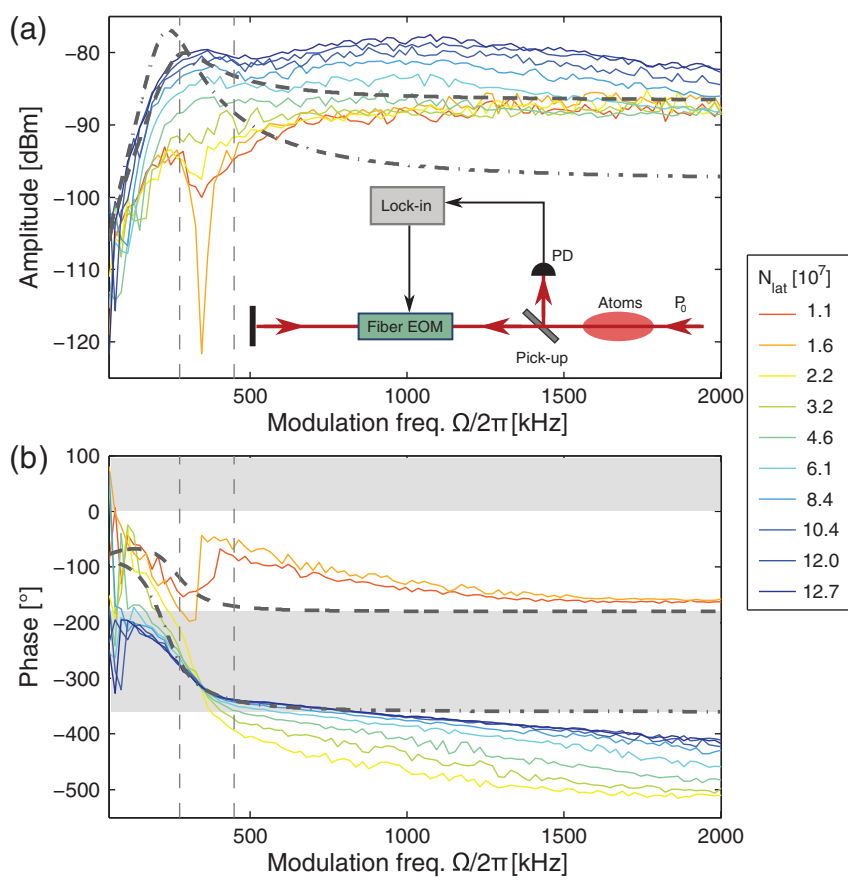

FIG. 3. Atomic backaction on the lattice light. (a) Amplitude (electrical power) and (b) phase of atomic backaction versus modulation frequency $\Omega$. Dashed vertical lines: Membrane frequency $\Omega_{m} / 2 \pi=276 \mathrm{kHz}$ and frequency of the atoms in the center of the trap $\Omega_{a}(0) / 2 \pi=450 \mathrm{kHz}$. Shaded areas: region in which the coupled system can become unstable if the coupling is strong enough. Inset in (a): Measurement setup. PD: photodiode. Thick dashed (dashed-dotted) lines: Behavior expected from the one-BS model (two-BS model) for $N=3 \times 10^{8}, \quad \Omega_{a}=2 \pi \times 275 \mathrm{kHz}, \quad \Gamma_{a}=2 \pi \times 150 \mathrm{kHz}$, and $R=0.06$. The modeled amplitudes are scaled down by $43 \mathrm{~dB}$ to adjust to the data.

In summary, we observed for the first time lightmediated atom-atom interactions in a free-space optical lattice giving rise to collective atomic oscillations and lattice instabilities. In our experiment these effects are enhanced by coupling the atoms to a distant dielectric membrane oscillator, which at the same time serves as a sensitive probe for the light-mediated collective atomic motion. The instabilities and collective dynamics in this hybrid system are described well by a model adapted from Asboth et al. [10].

Our experiment shows that substantial light-mediated atom-atom interactions can arise in free-space optical lattices in a regime of large atom numbers, moderate atom-light detuning, and asymmetric driving of the lattice. This offers new possibilities for the study of many-body physics, such as the spontaneous crystallization of atoms and light into a structure that features phononlike excitations and bears similarities to a supersolid [8]. Moreover, our results are relevant for the development of hybrid atomoptomechancial systems in the quantum regime [11-15]. The configuration studied in our experiment has been 
proposed for ground-state cooling of mechanical oscillators in regimes where purely optomechanical techniques fail [16-18]. Variants of the setup have been suggested for the generation of nonclassical vibrational states of mechanical oscillators [20,21]. In both cases, light-mediated atom-atom interactions have to be taken into account. Finally, such interactions could be harnessed to study nonequilibrium quantum phase transitions [22].

We thank Niels Lörch, Christoph Bruder, Andreas Jöckel, and Dan Stamper-Kurn for useful discussions. This work was supported by the project "Modular mechanical-atomic quantum systems" (MODULAR) of the European Research Council (ERC).

*Formally known as née Faber.

aline.faber@unibas.ch

${ }^{\dagger}$ Present address: Institut für Quantenmaterie and Center for Integrated Quantum Science and Technology $\left(\mathrm{IQ}^{\mathrm{ST}}\right)$, Universität Ulm, 89069 Ulm, Germany. *philipp.treutlein@unibas.ch

[1] I. Bloch, J. Dalibard, and W. Zwerger, Rev. Mod. Phys. 80, 885 (2008).

[2] D. Jaksch and P. Zoller, Ann. Phys. (Amsterdam) 315, 52 (2005).

[3] A. D. Ludlow, M. M. Boyd, J. Ye, E. Peik, and P. O. Schmidt, Rev. Mod. Phys. 87, 637 (2015).

[4] I. H. Deutsch, R. J. C. Spreeuw, S. L. Rolston, and W. D. Phillips, Phys. Rev. A 52, 1394 (1995).

[5] G. Birkl, M.Gatzke, I. H. Deutsch, S. L. Rolston, and W. D. Phillips, Phys. Rev. Lett. 75, 2823 (1995).

[6] M. Weidemüller, A. Görlitz, T.W. Hänsch, and A. Hemmerich, Phys. Rev. A 58, 4647 (1998).

[7] S. Ostermann, M. Sonnleitner, and H. Ritsch, New J. Phys. 16, 043017 (2014).

[8] S. Ostermann, F. Piazza, and H. Ritsch, Phys. Rev. X 6, 021026 (2016).

[9] J. K. Asbóth, H. Ritsch, and P. Domokos, Phys. Rev. Lett. 98, 203008 (2007).

[10] J. K. Asbóth, H. Ritsch, and P. Domokos, Phys. Rev. A 77, 063424 (2008).

[11] D. Hunger, S. Camerer, M. Korppi, A. Jöckel, T. W. Hänsch, and P. Treutlein, C.R. Phys. 12, 871 (2011).

[12] P. Treutlein et al., in Cavity Optomechanics, edited by M. Aspelmeyer, T. Kippenberg, and F. Marquardt (Springer, Berlin Heidelberg, 2014), pp. 327-351.
[13] S. Camerer, M. Korppi, A. Jöckel, D. Hunger, T. W. Hänsch, and P. Treutlein, Phys. Rev. Lett. 107, 223001 (2011).

[14] A. Jöckel, A. Faber, T. Kampschulte, M. Korppi, M. T. Rakher, and P. Treutlein, Nat. Nanotechnol. 10, 55 (2015).

[15] C. B. Møller, R. A. Thomas, G. Vasilakis, E. Zeuthen, Y. Tsaturyan, K. Jensen, A. Schliesser, K. Hammerer, and E. S. Polzik, arXiv:1608.03613.

[16] K. Hammerer, K. Stannigel, C. Genes, P. Zoller, P. Treutlein, S. Camerer, D. Hunger, and T. W. Hänsch, Phys. Rev. A 82, 021803 (2010).

[17] B. Vogell, K. Stannigel, P. Zoller, K. Hammerer, M. T. Rakher, M. Korppi, A. Jöckel, and P. Treutlein, Phys. Rev. A 87, 023816 (2013).

[18] J. S. Bennett, L. S Madsen, M. Baker, H. RubinszteinDunlop, and W. P. Bowen, New J. Phys. 16, 083036 (2014).

[19] K. Hammerer, M. Aspelmeyer, E. S. Polzik, and P. Zoller, Phys. Rev. Lett. 102, 020501 (2009).

[20] A. Carmele, B. Vogell, K. Stannigel, and P. Zoller, New J. Phys. 16, 063042 (2014).

[21] B. Vogell, T. Kampschulte, M. T. Rakher, A. Faber, P. Treutlein, K. Hammerer, and P. Zoller, New J. Phys. 17, 043044 (2015).

[22] N. Mann, M. R. Bakhtiari, A. Pelster, and M. Thorwart, arXiv:1710.10257.

[23] H. Ritsch, P. Domokos, F. Brennecke, and T. Esslinger, Rev. Mod. Phys. 85, 553 (2013).

[24] A. M. Jayich, J. C. Sankey, B. M. Zwickl, C. Yang, J. D. Thompson, S. M. Girvin, A. A. Clerk, F. Marquardt, and J G. E. Harris, New J. Phys. 10, 095008 (2008).

[25] See Supplemental Material at http://link.aps.org/ supplemental/10.1103/PhysRevLett.120.073602 for detailed description of the experimental setup, preparation of the atomic ensemble, calibration of the membrane displacement, determination of the optomechanical damping rate, transfer matrix model, modeling the atomic backaction, and influence of additional delay.

[26] R. Grimm, M. Weidemüller, and Y. B. Ovchinnikov, Adv. At. Mol. Opt. Phys. 42, 95 (2000).

[27] M. Aspelmeyer, T. J. Kippenberg, and F. Marquardt, Rev. Mod. Phys. 86, 1391 (2014).

[28] The curve with one BS saturates when $g_{N} \approx \Gamma_{a}$ and decreases for larger $N_{\text {lat }}$ because also a single BS modifies the light field configuration. However $\Gamma_{\text {tot }}$ remains positive for all $N_{\text {lat }}$ in this case.

[29] G. F. Franklin, J. D. Powell, and A. Emami-Naeini, Feedback Control of Dynamic Systems (Pearson, London, 2015). 